\title{
Short-period Variations of 28 CMa during 1998/9
}

\author{
L.A. Balona \\ South African Astronomical Observatory, PO Box 9, Observatory 7935, \\ Cape Town, South Africa \\ D. James
}

School of Physics and Astronomy, University of St. Andrews, Scotland

\begin{abstract}
The Be star $28 \mathrm{CMa}$ was one of the first periodic Be stars to be discovered and shows very large line profile variations with a period of $1.37 \mathrm{~d}$. Recently, it has been shown that the line profile and light variations can be modeled by a patch of gas suspended above the photosphere. We present echelle observations of the $\mathrm{H} \beta$ and $\mathrm{H} \gamma$ line and several helium and metal absorption lines. We show that the radial velocity variations of these lines are unchanged since they were first observed two decades ago. We also examined several emission lines of Fe II and show that they do not partake of the periodic variation. We attribute the periodic variations of the lines formed close to the photosphere to a co-rotating cloud, whereas the Fe II emission lines are formed in the circumstellar disk outside the co-rotating radius.
\end{abstract}

\section{Introduction}

Periodic line profile and radial velocity variations in $28 \mathrm{CMa}$ were discovered by Baade (1982) who interpreted them in terms of nonradial pulsation (NRP). The profile variations of $\mathrm{He}$ I 6678 were examined in detail by Balona, Aerts \& Štefl (1999), who were unable to fit the line profiles with NRP. The problem is that the very large radial velocity amplitude $\left(\approx 80 \mathrm{~km} \mathrm{~s}^{-1}\right)$ and very small light amplitude ( $<0.005 \mathrm{mag}$ ) seems to be incompatible with NRP or starspots. However, Balona et al. (1999) did not consider the case where the period is negative. Maintz et al. (1999) find a physically meaningful NRP solution in such a case: an $\ell=2$ retrograde mode in which the period of the star is shorter than the apparent period of the mode.

On the assumption that the variations are caused by a circular patch with the same temperature as the photosphere, but a much smaller intrinsic line width, Balona, Aerts \& Stefl (1999) were able to obtain a good match with the line profiles and light variations, a conclusion which is still valid in spite of the finding by Maintz et al. (1999). This "patch" model has now been proposed in three other stars: $\eta$ Cen (Balona 1999), $\zeta$ Tau (Balona \& Kaye 1999) and $\mu$ Cen (Balona, James, Lawson \& Shobbrook 1999). It seems that the patch is actually a cloud of gas near the photosphere co-rotating with the star. This idea is the basis for a revival of an old explanation for the periodic Be stars. 



Figure 1. Radial velocities of some helium and metal lines (in $\mathrm{km} \mathrm{s}^{-1}$ ) phased with a period of $1.37202 \mathrm{~d}$. The radial velocity is defined as the position of minimum line intensity of the line profile.

Štefl et al. (1998) report the occurrence of a transient period with $\mathrm{P}=$ $1.49 \mathrm{~d}$ which is sometimes visible in the wings of the helium lines, in the Si II and $\mathrm{Mg}$ II lines and dominates in $\mathrm{H} \alpha$ and $\mathrm{H} \beta$. This period is also present in the photometry (Štefl, Aerts \& Balona 1999). The observations described here were obtained with a view to monitoring the periods in the lines and to look for other clues which might clarify the nature of Be stars.

\section{Observations}

The data were obtained with the SAAO echelle spectrograph (GIRAFFE) attached to the $1.9-\mathrm{m}$ telescope. The resolving power is approximately 35000 . A total of 67 spectra were obtained during observing runs in 1998, March, September, December and in 1999, March. The radial velocity of the point of minimum intensity of the line was determined by fitting a quadratic function.

Fig. 1 shows the velocity curves of several helium and metal lines. The behaviour of these lines is essentially unchanged since the observations of Baade (1982). The radial velocities can be matched to those obtained in 1994 and 1996 by the following ephemeris:

$$
T=2449660.9112+1.37202 n
$$

where $T$ is the time of maximum radial velocity. There is no indication of the transient period in these data or in the radial velocity of Si II 5056.

In Fig. 2 we show the variations in the ratio of the strengths of the violet and red emission peaks in $\mathrm{H} \beta$ and $\mathrm{H} \gamma$ (the $\mathrm{V} / \mathrm{R}$ ratio). Periodic $\mathrm{V} / \mathrm{R}$ variations in the hydrogen lines were originally found by Baade (1982). The period is the 

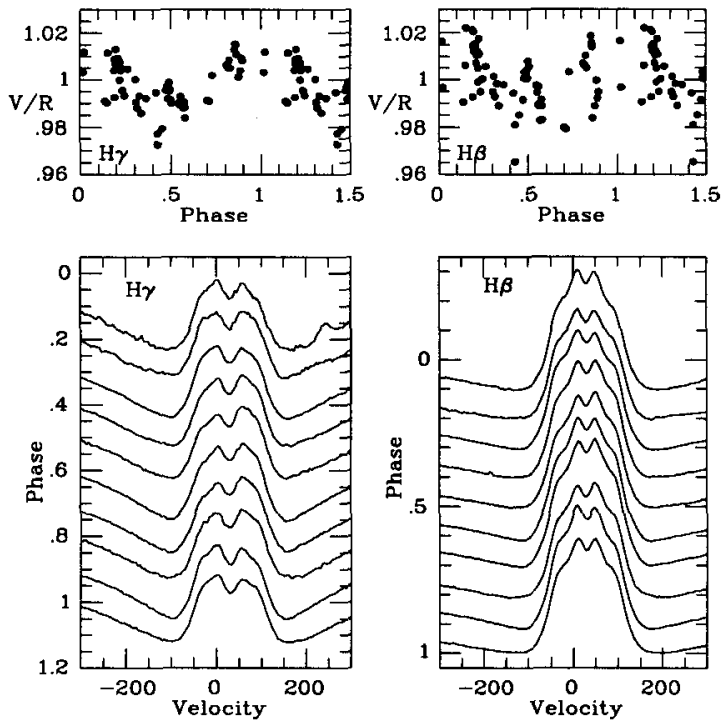

Figure 2. Line profiles of the $\mathrm{H} \beta$ and $\mathrm{H} \gamma$ lines in ten phase bins showing periodic $V / R$ variations. The panel above the line profiles shows the $V / R$ ratio as a function of phase.

same as found in the photospheric lines and is probably different in nature from the long-term $\mathrm{V} / \mathrm{R}$ variations found in other Be stars. We looked for, but were not able to find, any other significant frequencies. The transient period of 1.49 $\mathrm{d}$ does not seem to be present.

\section{Emission lines}

Emission lines of Fe II are present in all our spectra except during the last run (JD 2451261 - 2451274). They all show a central absorption which seems to vary from time to time. The lines are weak, so it is difficult to determine any systematic effects. During the last run these lines are either absent or present as very weak single-peak emission lines. In Fig. 3 we show co-added profiles of the Fe II 5317 line for all spectra excluding the last run. They are phased with the 1.37-d period. There seems to be no obvious phase dependence.

\section{Discussion}

The periodic variations in $28 \mathrm{CM}$ a have been quite stable over the last decade or even longer. There is no evidence of the large changes in amplitude that occur in most Be stars. We did not detect the transient $1.49-\mathrm{d}$ period during the time of our observations.

We attribute the periodic variations in this star to an approximately circular cloud of gas suspended above the photosphere at an intermediate latitude 


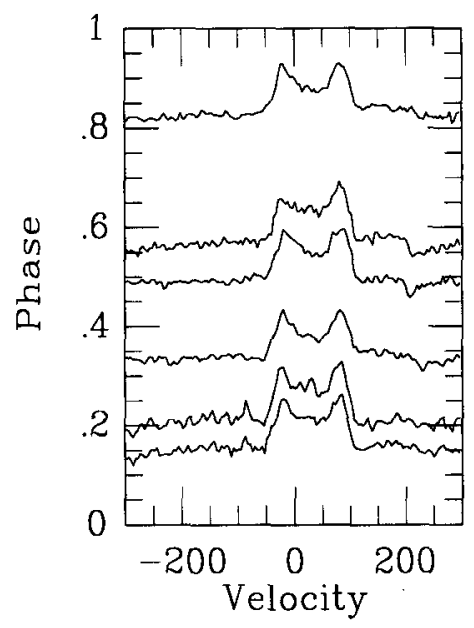

Figure 3. Line profiles of Fe II 5317 phased with a period of $1.37202 \mathrm{~d}$.

(Balona, Aerts \& Štefl 1999). The periodic $\mathrm{V} / \mathrm{R}$ variations in $\mathrm{H} \beta$ and $\mathrm{H} \gamma$ can be understood as the underling absorption profile distorting the circumstellar emission components. The Fe II emission lines presumably originate in a region far from the central star and do not show the periodic variations associated with the co-rotating material.

The transient period only appears in lines of low excitation, indicating that it originates in the inner circumstellar disk. The cause of the periodicity is unknown. The thermal timescale in the upper atmosphere or inner disk is at least two orders of magnitude shorter than the period. This seems to rule out a trapped pulsation mode as an explanation. Also, g-modes are evanescent in regions above the photosphere which rules out the possibility of a node at the photospheric level. On these general grounds, NRP is most unlikely to be responsible for the transient period.

\section{References}

Baade, D. 1982, A\&A 105, 65

Balona, L.A. 1999, MNRAS 306, 407

Balona, L.A., Kaye, A.B. 1999, ApJ, in press

Balona, L.A., Aerts C., Štefl, S. 1999, MNRAS 305, 519

Balona, L.A., James, D., Lawson, W.A., Shobbrook, R.R. 1999, these Proceedings.

Štefl, S., Baade, D., Rivinius, Th., Stahl, O., Wolf, B., Kaufer, A. 1998, in A Half-century of Stellar Pulsation Interpretations, eds. P.A. Bradely \& J.A. Guzik, ASP Conference Series 135, p348.

Štefl, S., Aerts, C., Balona, L.A. 1999, MNRAS 305, 505

Maintz, M., Wolf, B., Rivinius, Th., Tubbesing, S. 1999, these Proceedings. 